\title{
ANÁLISIS DE COSTO - OPORTUNIDAD DEL USO DE COPROSCÓPICO EN DIARREA AGUDA EN MENORES DE 5 AÑOS
}

\author{
ELIANA LÓPEZ BARÓN¹, JUAN CARLOS MORALES JIMÉNEZ², \\ FABIO RODRÍGUEZ MORALES ${ }^{3}$
}

\begin{abstract}
${ }^{1} \mathrm{Md}$. Pediatra, Universidad de La Sabana. Departamento de Pediatría, Facultad de Medicina, Universidad de La Sabana. Hospital de Vista Hermosa, Bogotá.

${ }^{2}$ Md. Pediatra, Universidad de La Sabana. Departamento de Pediatría, Facultad de Medicina, Universidad de La Sabana. Hospital de Vista Hermosa, Bogotá.

${ }^{3}$ Pediatra, Epidemiólogo. Docente Universidad de La Sabana. Departamento de Pediatría, Facultad de Medicina, Universidad de La Sabana. Hospital de Vista Hermosa, Bogotá.

Correspondencia: Eliana López Barón. Universidad de La Sabana, Facultad de Medicina. Campus del Puente del Común, Km. 7, Autopista Norte de Bogotá. Chía, Cund. Colombia

E-mail: elianaloba@unisabana.edu.co.
\end{abstract}

Recibido: 1 Enero 2016 Aceptado: 7 Junio 2016

\section{RESUMEN}

Objetivo: Analizar el uso de coproscópico de rutina, en menores de 5 años con diarrea aguda, en un Hospital de primer nivel de Bogotá y evaluar la mejor forma de asignar los recursos usados en esta intervención a una alternativa que produzca mayor beneficio.

Métodos: Revisión de la literatura en diarrea aguda y utilidad del coproscópico. Recopilación de datos de consultas por diarrea en niños de 0-5 años y coproscópicos realizados entre octubre de 2011 y febrero de 2012 en Hospital de Bogotá, con selección de aquellos sugestivos de enfermedad enteroinvasiva, cálculo del costo de su uso y estimación del costo-oportunidad en realización de programas que reduzcan la morbimortalidad en menores de 5 años.

Resultados: La principal etiología de la enfermedad diarreica es viral. Más de 5 leucos/campo tiene una sensibilidad y especificidad adecuada que no supera la valoración clínica. Se solicitó coproscópico en $44.8 \%$; el $14.6 \%$ sugerían enfermedad enteroinvasiva. El $30.2 \%$ de los pacientes no requerían coproscópico y su costo corresponde a $\$ 5.266 .400$.

Conclusiones: La utilidad del coproscópico es baja y no mejora la probabilidad pretest de diarrea enteroinvasiva, es necesario abordar la el análisis de costo-oportunidad para la distribución correcta de los recursos en intervenciones que reduzcan la morbimortalidad.

Palabras claves: Diarrea, Leucocitos fecales, Coproscópico. 


\title{
COST-OPPORTUNITY ANALYSIS OF THE USE OF FECAL SCREENING TEST IN ACUTE DIARRHEA IN CHILDREN UNDER 5 YEARS OLD
}

\begin{abstract}
Objective : To analyze the use of routine fecal screening test in children under 5 years old, with acute diarrheal disease in a primary care level hospital in Bogota and evaluate how best the resources used in this intervention can be allocated to an alternative one, that produces greater benefit .

Methods: Review of the literature on acute diarrhea and utility of fecal screening test. Data collection of diarrhea consultations in children under 5 years old and fecal screening test done between October 2011 and February 2012 in a Bogota's Hospital. Selecting those one, which diagnoses enteroinvasive diarrhea and costing of routine use, and estimated opportunity-cost in programs to reduce morbi-mortality in children under 5 years old.

Results: The main etiology of diarrhea is viral. More than 5 WBCs / field has adequate sensitivity and specificity, but the clinical assessment is better on detection of enteroinvasive disease. Fecal screening test was requested in 44.8 $\% ; 14.6 \%$ was suggested enteroinvasive disease. The resources used in $30.2 \%$ of patients did not require the test match to US $\$ 2.633$.

Conclusions: The utility of Fecal screening test is low and does not imprve the pretest probability of enteroinvasive diarrhea. Is necessary to address the cost-opportunity analysis for the proper distribution of resources in intervetions that reduce the morbi-mortality.
\end{abstract}

Keywords: Diarrhea, Fecal test, Stool analysis.

\section{ANÁLISE CUSTO-OPORTUNIDADE DO USO DO TESTE DE VERIFICAÇÃO FECAL NA DIARREIA AGUDA EM CRIANÇAS MENORES DE 5 ANOS}

\section{RESUMO}

Objetivo: Analisar o uso do teste rotineiro de triagem fecal em crianças menores de 5 anos com doença diarréica aguda em um hospital de atenção primária em Bogotá e avaliar a melhor forma de alocação dos recursos utilizados nessa intervenção para uma alternativa que produza maior benefício.

Métodos: Revisão da literatura sobre diarréia aguda e utilidade do teste de triagem fecal. Coleta de dados de consultas de diarréia em crianças menores de 5 anos e teste de triagem fecal realizado entre outubro de 2011 e fevereiro de 2012 em um hospital de Bogotá. Selecionar aquelas que diagnosticam diarréia entero-invasiva e custo de uso rotineiro e custo de oportunidade estimado em programas para reduzir a morbi-mortalidade em crianças menores de 5 anos.

Resultados: A principal etiologia da diarréia é viral. Mais de $5 \mathrm{WBCs} /$ campo tem sensibilidade e especificidade adequadas, mas a avaliação clínica é melhor na detecção de doença enteroinvasiva. O teste de triagem fecal foi solicitado em 44,8\%; 14,6\% foi sugerida doença enteroinvasiva. Os recursos utilizados em 30,2\% dos pacientes não necessitaram do teste correspondente a US \$2,633.

Conclusões: A utilidade do teste de triagem Fecal é baixa e não melhora a probabilidade pré-teste de diarréia enteroinvasiva. É necessário abordar a análise custo-oportunidade para a distribuição adequada de recursos em intervenções que reduzam a morbi-mortalidade.

Palavras-chave: Diarrea, Exame fecal, Análise de fezes . 


\section{Introducción}

La diarrea es un síntoma común de infección del tracto gastrointestinal, que puede ser producida por microorganismos virales, bacterianos o protozoarios; en países en desarrollo, la principal etiología es viral, estimándose que el rotavirus provoca 111 millones de episodios de gastroenteritis al año, 2 millones de hospitalizaciones y entre 352000 y 592000 muertes(1); en Colombia contamos con cifras similares, con el $57 \%$ de la etiología en enfermedad diarreica en niños menores de 5 años atribuida al rotavirus(2). En frecuencia le siguen bacterias como Escherichia coli, Shigella, Campylobacter jejuni y Salmonella $(3,4)$, y en Colombia, otros patógenos como $E$. histolytica y Giardia $(5,6)$, estos últimos más frecuentes en zonas con condiciones de vida insalubres (3). Los mecanismos de acción de los patógenos involucrados incluyen respuesta inflamatoria que en el caso de las bacterias enteroinvasivas involucra activación y quimiotaxis de leucocitos polimorfonucleares y se expresa en deposiciones con moco y sangre, leucocitos en las heces o presencia de lactoferrina fecal (7).

La enfermedad diarreica es la segunda causa de mortalidad en niños menores de 5 años y una causa muy importante de desnutrición infantil $(3,8)$. A nivel mundial tiene una incidencia de 2.5 billones de casos al año, siendo mayor en países localizados en África y Asia y una tasa de mortalidad en niños menores de 5 años del $15 \%$, superado solamente por la neumonía $(9,4)$. En las Américas y en Colombia el panorama no es diferente, con una carga de mortalidad del $7 \%$ para las Américas y un $4 \%$ para Colombia, en Colombia esto representa 7,34 muertes/100 000 niños menores de 5 años, $(8,9)$; con una marcada inequidad geográfica, siendo mayores estas tasas en departamentos como Chocó, Guajira, Amazonas, Cauca, Risaralda y Cesar. En Bogotá se encuentra una tasa de mortalidad por EDA de 1.01 muertes por 100000 niños, sin embargo esta presenta variaciones en las diferentes localidades, con mayores tasas es zonas como la localidad de Ciudad Bolívar, que es de 3.08 muertes por 100000 habitantes (10).

El diagnóstico de la diarrea se basa en los hallazgos clínicos y para el inicio del tratamiento no es necesario la realización del examen microscópico de materia fecal ni el cultivo (4). Se ha evaluado la confiabilidad de marcadores en materia fecal que identifiquen diarreas secundarias a procesos invasivos bacterianos o parasitarios y que justifiquen el inicio de antibioticoterapia, observando el comportamiento de leucocitos, glóbu- los rojos, sangre oculta y lactoferrina en materia fecal; demostrando que las mejores pruebas son la lactoferrina, seguida de la sangre oculta y la menos adecuada es el conteo de leucocitos en materia fecal, que pueden aumentar su efectividad si se acompañan de los datos clínicos(11), con sensibilidad del $50 \%$, especificidad del $83 \%$, para leucocitos fecales (punto de corte $>5 /$ campo), y sensibilidad de $22 \%$, especificidad de $91 \%$ y para eritrocitos fecales (punto de corte $>10 /$ campo).(12). Se ha reportado que de los pacientes con rotavirus, el 16\% muestra leucocitos en materia fecal y el 39\% sangre oculta (13). Estudios previos muestran un abuso en la solicitud de coproscópico en Bogotá durante la evaluación del niño con diarrea. De acuerdo con los resultados de este trabajo se acude a esta prueba en más del $80 \%$ de los casos de diarrea en menores de cinco años. Por otra parte, el resultado obtenido en el coproscópico, modifica la conducta terapéutica del médico en apenas una tercera parte de los casos, cambios que en el $15 \%$ de las circunstancias no parecían justificados pues el coproscópico estaba dentro de lo normal (14). Klein y colaboradores en su estudio demuestran otros datos clínicos que dan porcentajes de sensibilidad de hasta el $85 \%$ para esta etiología (historia de viajes a zonas de prevalencia, fiebre, más de 10 deposiciones en 24 horas, sangre en las heces); además se demostró que el juicio médico tiene una sensibilidad del $76 \%$ y especificidad del $65 \%$ para la sospecha diagnóstica de diarrea bacteriana (15).

El promedio total del costo por episodio de diarrea en Estados Unidos es de US \$289 (16). Teniendo en cuenta las limitaciones de recursos en salud, con la consiguiente necesidad de control de los mismos y su alto costo, se realizará un análisis de costo oportunidad que busca identificar la mejor forma de asignar los recursos teniendo en cuenta que una vez que se decide asignar recursos a un programa o actividad se pierde la oportunidad de asignarlos a otro; de esta forma se busca que los recursos sean asignados a la alternativa que produzca el mayor beneficio, que pueden ser medidos por los indicadores de calidad en salud. El costo de oportunidad de una intervención se define como el valor de los recursos que esta intervención utiliza, si ellos hubieran sido asignados a su uso alternativo más valioso (17).

\section{Materiales y métodos}

Se realizó un estudio de costo-oportunidad tomando como muestra todos los casos de consultas por enfermedad diarreica aguda en niños de 0 a 5 años que asis- 
tían al servicio de urgencias registrados en la base de datos de historia clínica electrónica del hospital Vista Hermosa para el período de tiempo comprendido entre los meses de octubre de 2011 a febrero de 2012.

Como criterio de inclusión de la muestra de estudio se realizó filtro a la información obtenida del hospital para el período de tiempo mencionado incluyendo los datos de niños consultantes con edad menor de 5 años cuyo diagnóstico correspondiera al de enfermedades infecciosas intestinales utilizando para su identificación el código CIE-10 (A00-A09), y seleccionando únicamente los casos en los que se había solicitado el examen diagnóstico (coproscópico) excluyendo aquellos en los que no se había solicitado el estudio paraclínico, en quienes se hubiese solicitado pero no se logró recolección de la muestra, en quienes se solicitó muestra, esta fue recolectada pero no se contaba con la información del laboratorio o cuyo origen de solicitud fuera consulta externa; del total de estos registros obtenidos y para efectos del análisis de costo-oportunidad se consideraron útiles en el diagnóstico de enfermedad diarreica aguda, aquellos coproscópicos cuyo resultado tuviera los siguientes criterios: leucocitos fecales $>5$ por campo; eritrocitos fecales $>5$ por campo, quistes o trofozoitos de Giardia o trofozoitos de E. Histolytica (12), teniendo en cuenta la sensibilidad del 50\%, especificidad del 83\%, para leucocitos fecales (punto de corte $>5 /$ campo), y sensibilidad de $22 \%$, especificidad de $91 \%$ y para eritrocitos fecales (punto de corte $>10 /$ campo), encontrada en estudios previos (12).

Con los datos obtenidos, se cuantificó el costo del número total de coproscópicos solicitados y de los diagnósticos para enfermedad diarreica aguda, tomando como referencia el valor de un coproscópico en el laboratorio del Hospital de Vista Hermosa, en pesos colombianos que corresponde a \$23.200 (COP).

Con los valores obtenidos, se estima el costo de oportunidad que corresponde a lo que se puede hacer si los recursos que se invierten en la solicitud de coproscópicos se destinan a otras intervenciones. Como alternativas se escogieron acciones que si tienen efectividad demostrada en enfermedad diarreica aguda por la Organización Mundial de la Salud como como la provisión de zinc y en la disentería el uso de ácido nalidíxico; así mismo el uso de urocultivo considerado como el estándar de oro para diagnóstico de infección de vías urinarias en niños, intervenciones que no son cubiertas por el sistema de salud actual en el primer nivel de complejidad; igual- mente se considera alternativa de uso de estos recursos la capacitación e implementación de programas que incentivan intervenciones que han demostrado disminución de la morbilidad infantil como la lactancia materna, la prevención de accidentes en el hogar, prevención del maltrato, pautas de crianza y salud oral, entre otras.

\section{Resultados}

En el hospital de Vista Hermosa, centro de referencia de la localidad de Ciudad Bolívar, la enfermedad diarreica aguda generó para el año 2011, un total de 2298 consultas en niños menores de 5 años, de los cuales se hospitalizaron 382 pacientes. La prevalencia de la enfermedad diarreica aguda en el hospital de Vista Hermosa de la localidad de Ciudad Bolivar fue de 3.5\%, teniendo en cuenta la población entre $0-5$ años en la localidad de Ciudad Bolívar, que es de 65 580, según estadísticas del DANE para el año 2011 y el número de niños que consultaron por EDA entre marzo de 2011 y febrero de 2012.

De las 2298 consultas al año por EDA, un $81.1 \%$ ingresa por el servicio de urgencias, mientras el $18.9 \%$ restante lo hace por el servicio de consulta externa. De estas consultas por EDA, un $9.1 \%$ es hospitalizado. (Tabla 1). El promedio de edad, de los niños a los que se les realizó coproscópico es de 2.4 años ( 0 - 5 años).

A partir de los datos descritos anteriormente se calculó el número de coproscópicos realizados entre octubre de 2011 y febrero de 2012, de estos se tomaron los que se consideraron diagnósticos en enfermedad diarreica aguda invasiva y se estimó su costo en pesos colombianos, de acuerdo con su valor unitario en el Hospital de Vista Hermosa de \$23.200 (COP) (tabla 2 y 3, gráfica 1).

La tabla 4, muestra el número de consultas por enfermedad diarreica aguda entre los meses de octubre de 2011 y febrero de 2012 correspondiente a 751 consultan; al $44.8 \%$ de los pacientes que asistieron a estas consultas se les solicitó coproscópico, de los cuales solo un $14.6 \%$ mostró un resultado compatible con EDA enteroinvasiva. Los recursos empleados en el $30.2 \%$ de los pacientes que no requerían coproscópico, que corresponde a \$5.266.400 (COP) representa el costo de oportunidad perdido en otras alternativas demostradas efectivas en reducir la morbimortalidad infantil. El cálculo de este costo-oportunidad es mostrado en la tabla 5 , donde indica la inversión en programas e intervenciones que se podría realizar con este monto de dinero. 


\section{Tablas y gráficos}

Tabla 1. Consultas por enfermedad diarreica aguda entre marzo de 2011 - febrero 2012 en los servicios de urgencias, consulta externa (CE) y los pacientes hospitalizados por enfermedad diarreica aguda.

\begin{tabular}{|l|c|c|c|c|c|c|c|c|c|c|c|c|c|}
\hline & $\begin{array}{c}\text { mar- } \\
\mathbf{1 1}\end{array}$ & $\begin{array}{c}\text { abr- } \\
\mathbf{1 1}\end{array}$ & $\begin{array}{c}\text { may- } \\
\mathbf{1 1}\end{array}$ & $\begin{array}{c}\text { jun- } \\
\mathbf{1 1}\end{array}$ & $\begin{array}{c}\text { jul- } \\
\mathbf{1 1}\end{array}$ & $\begin{array}{c}\text { ago- } \\
\mathbf{1 1}\end{array}$ & $\begin{array}{c}\text { sep- } \\
\mathbf{1 1}\end{array}$ & $\begin{array}{c}\text { oct- } \\
\mathbf{1 1}\end{array}$ & $\begin{array}{c}\text { nov- } \\
\mathbf{1 1}\end{array}$ & $\begin{array}{c}\text { dic- } \\
\mathbf{1 1}\end{array}$ & $\begin{array}{c}\text { ene- } \\
\mathbf{1 2}\end{array}$ & $\begin{array}{c}\text { feb- } \\
\mathbf{1 2}\end{array}$ & $\begin{array}{c}\text { TOTAL } \\
\text { (n) }\end{array}$ \\
$\mathbf{( \% )}$
\end{tabular}

Tabla 2. Número de coproscópicos solicitados por mes y los considerados de diagnóstico en enfermedad entero invasiva.

\begin{tabular}{|l|c|c|c|c|c|}
\hline & oct-11 & nov-11 & dic-11 & ene-12 & feb-12 \\
\hline Solicitados & 75 & 85 & 58 & 56 & 63 \\
\hline Positivos para EDA entero invasiva & 24 & 22 & 18 & 22 & 24 \\
\hline
\end{tabular}

Criterios de enfermedad entero invasiva (leucocitos fecales $>5$ por campo; eritrocitos fecales $>5 /$ campo, quistes o trofozoitos de Giardia o trofozoitos de E. Histolytica).

Tabla 3. Costo de los coproscópicos solicitados mensuales y los útiles para EDA en 5 meses.

\begin{tabular}{|l|c|c|c|c|c|c|}
\hline & oct-11 & nov-11 & dic-11 & ene-12 & feb-12 & TOTAL \\
\hline SOLICITADOS & 75 & 85 & 58 & 56 & 63 & 337 \\
\hline Precio (COP) & 1.740 .000 & 1.972 .000 & 1.345 .600 & 1.299 .200 & 1.461 .600 & $\mathbf{7 . 8 1 8 . 4 0 0}$ \\
\hline Positivos EDA entero invasiva & 24 & 22 & 18 & 22 & 24 & 110 \\
\hline Precio (COP) & 556.800 & 510.400 & 417.600 & 510.400 & 556.800 & $\mathbf{2 . 5 5 2 . 0 0 0}$ \\
\hline
\end{tabular}

$\mathrm{COP}=$ Pesos colombianos

Tabla 4. Coproscópicos realizados y costo de oportunidad a 5 meses

\begin{tabular}{|l|c|c|c|c|c|c|c|}
\hline & oct-11 & nov-11 & dic-11 & ene-12 & feb-12 & Total & Promedio (\%) \\
\hline Consultas EDA & 146 & 107 & 104 & 169 & 225 & 751 & $150(100)$ \\
\hline Coproscópicos solicitados & 75 & 85 & 58 & 56 & 63 & 337 & $67.4(44.8)$ \\
\hline Precio (COP) & 1.740 .000 & 1.972 .000 & 1.345 .600 & 1.299 .200 & 1.461 .600 & 7.818 .400 & 110 \\
\hline Coproscópicos diagnóstico EDA & 24 & 22 & 18 & 22 & 24 & $22(14.6)$ \\
\hline Precio (COP) & 556.800 & 510.400 & 417.600 & 510.400 & 556.800 & 2.552 .000 & \\
\hline Costo de oportunidad & 51 & 63 & 40 & 34 & 39 & 227 & $45.4(30.2)$ \\
\hline Precio (COP) & 1.183 .200 & 1.461 .600 & 928.000 & 788.800 & 904.800 & 5.266 .400 & \\
\hline
\end{tabular}

\section{COP $=$ Pesos colombianos}


Tabla 5. Inversión del costo de oportunidad de 5 meses.

\begin{tabular}{|l|c|c|c|}
\hline INTERVENCIÓN/PROGRAMA & PRECIO (COP) & NÚMERO & TOTAL \\
\hline Sulfato de Zinc & 10.000 & 526 & 5.260 .000 \\
\hline Ácido Nalidíxico & 24.950 & 211 & 5.264 .450 \\
\hline Urocultivo & 60.000 & 87 & 5.220 .000 \\
\hline Programa de promoción y prevención & 1.053 .280 & 5 & 5.266 .400 \\
\hline
\end{tabular}

\section{$\mathrm{COP}=$ Pesos colombianos}

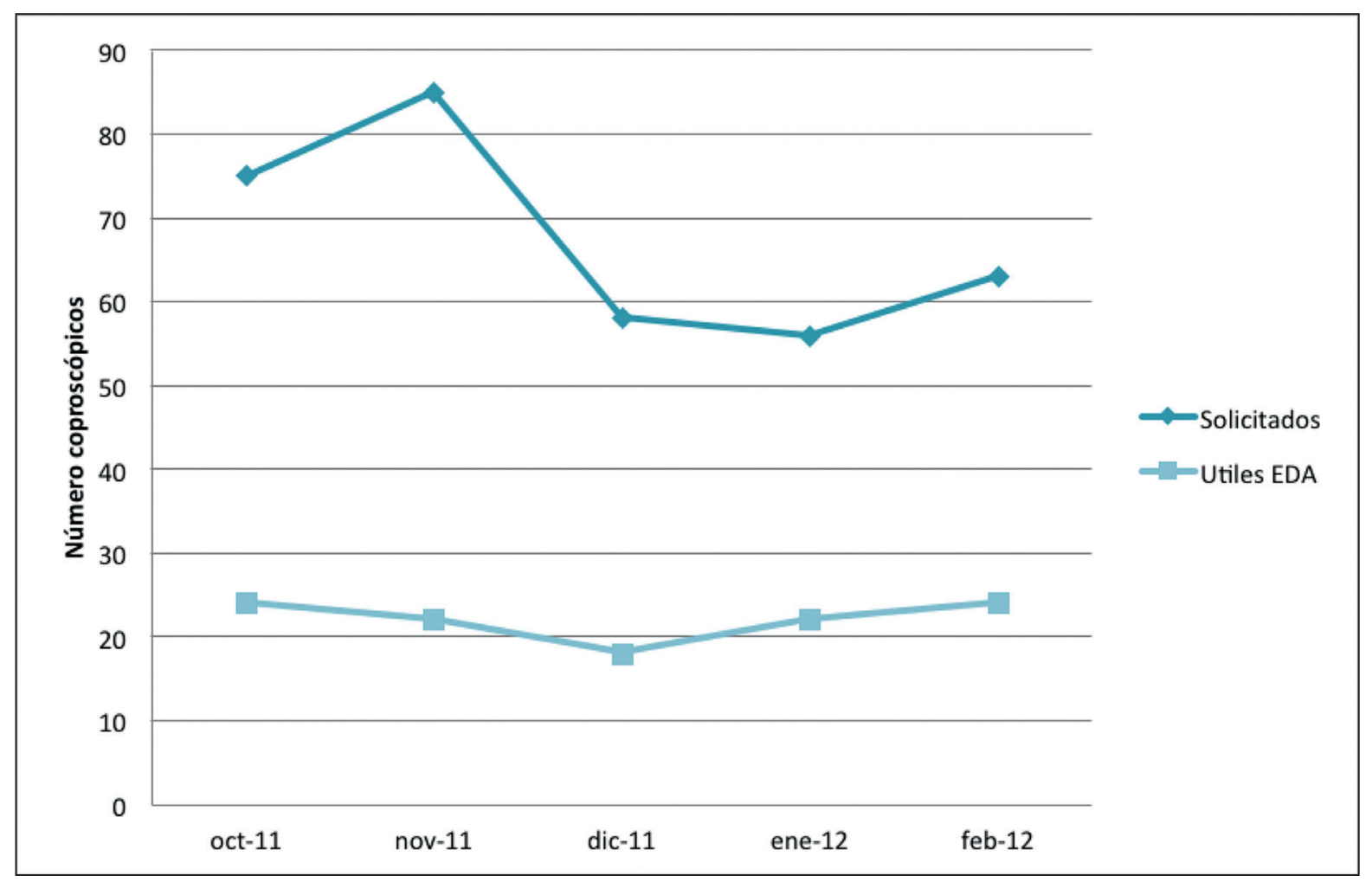

Gráfica 1. Coproscópicos solicitados y útiles para diagnóstico y manejo de EDA. Datos tomados en los meses de octubre de 2011 a febrero de 2012. 


\section{Discusión y conclusiones}

Este estudio de costo de oportunidad realiza una evaluación de la pertinencia de la toma de coproscópico en enfermedad diarreica aguda, dado que la literatura demuestra su etiología principalmente viral y su carácter autolimitado; usando los puntos de corte encontrados útiles para evaluar enfermedad diarreica enteroinvasiva, se demostró que es baja la utilidad de realizar este estudio en el hospital de Vista Hermosa dada la baja frecuencia de enfermedad enteroinvasiva bacteriana o parasitaria y la utilidad de los hallazgos clínicos para identificar esta etiología así como de los factores de riesgo del paciente que permitan sus sospecha, en estudio realizado por Briceño G, Vera J (2010) se concluye que cuando hay sospecha de diarrea de posible origen bacteriano en niños con factores como edad de 24 meses o menos, fiebre $\geq 39^{\circ} \mathrm{C}$ durante la enfermedad actual, historia de consumo de bebida o alimento presuntamente contaminado o contacto con alguna persona con infección invasiva intestinal, la presencia de 20 o más leucocitos en el coproscópico, el $\mathrm{pH} \geq 8$ de la materia fecal o la presencia de eritrocitos, no mostró asociación entre estos hallazgos individuales del coproscópico y la etiología bacteriana de la diarrea (18).

Basados en esto se realizó una evaluación económica de la oportunidad que se pierde al invertir dinero en la toma de este examen que no mejora la probabilidad pretest de tener o no una diarrea por patógeno enteroinvasivo como si lo hacen los datos de la historia clínica y que podría utilizarse en alternativas recomendadas por organizaciones mundiales que demuestran beneficios en la reducción de la morbimortalidad infantil como el uso de sales de zinc que disminuye la gravedad y duración de la EDA y el uso del ácido nalidíxico en cuadros de disentería, esto recomendado por la estrategia de Atención de Enfermedades Prevalentes de la Infancia (AIEPI) $(18,19)$, los cuales son intervenciones que no se encuentran en el plan obligatorio de salud actual. Otras alternativas a esto serían el uso de urocultivo como estándar de oro en el diagnóstico de infección de vías urinarias, patología que tiene una incidencia del $2 \%$ en niños menores de 5 años según estudios en un hospital de primer nivel de complejidad de Bogotá (Colombia) (19), y cuya utilidad se vería reflejada en evitar el uso indiscriminado de antibioticoterapia que además de presentar posibles efectos adversos, aumenta las tasas de resistencia bacteriana a antibióticos. Otra opción que se plantea, es el uso de estos recursos en programas que incentiven prácticas claramente estudiadas y recomendadas actualmente por la OMS, que lleven a disminuir la morbilidad infantil, estas son, incen- tivar la lactancia materna, evitar el maltrato, incentivar la salud oral y prevención de accidentes en el hogar.

En un sistema de salud donde los recursos son limitados, se tendrá necesariamente que abordar el estudio y la implementación de la evaluación económica, para la distribución correcta de los mismos; los estudios de costo de oportunidad se consideran como uno de los métodos más adecuados para este propósito.

\section{Referencias}

1. Parashar D, Hummelman EG, Bresse JS, et al. Global illness and deaths caused by rotavirus disease in children. Emerging Infect Dis. 2003; 9(5):565-72

2. Instituto Nacional de Salud. Informe Quincenal Epidemiológico Nacional 2003; 8(20):321-36.

3. Benguini Y, Bernal C, Figueroa D. Fisiología y etiología de las enfermedades diarreicas. En: Organización panamericana de la Salud. Manual de tratamiento de la diarrea en niños. Serie PALTEX para ejecutores de programas de salud No 48. 2008.pp 75 - 133.

4. UNICEF, Organización Mundial de la Salud. Diarrhea:Why children are still dying and what can be done. 2009.

5. Manrique-Abril FG, Billon Y Tigne D, Bello SE, Ospina JM. Agentes causantes de Diarrea en Niños Menores de 5 Años en Tunja, Colombia. Revista de Salud Pública [revista en Internet] 2006; 888-97. Disponible en: http:// www.redalyc.org/articulo id $=42280108$.

6. Bernal C, Zapata CT, Durango HE, Álvarez CM. Agentes etiológicos de diarrea en niños atendidos en la Unidad de Capacitación para el Tratamiento de la Diarrea del Hospital Universitario San Vicente de Paúl de Medellín. Infectio 2002; 6(4): 204-211

7. Carreazo NY, Ugarte K, Huicho L. Leucocitos fecales en niños con diarrea aguda: ¿momento de reconsiderar la utilidad clínica de la prueba?. Rev. gastroenterol. Perú, jul - sep. 2011, vol.31, no.3, p.216-223.

8. Organización Mundial de la Salud. Estadísticas sanitarias mundial, 2011. Disponible en: http://www.who.int/whosis/whostat/2011/es/index.html

9. Black RE, Cousens S, Johnson HL, et al. Global, regional, and national causes of child mortality in 2008: a systematic analysis. Lancet 2010; 375: 1969-87

10. Equipo de Análisis de Situación en Salud, ASIS. Análisis de Situación en Salud de Ciudad Bolivar, 2011.

Disponible en www.hospitalvistahermosa.gov.co

11. Huicho L, Campo M, Rivera J, et al. Fecal screening tests in the approach to acute infectious diarrhea: a scientific overview. Pediatr. Infect. Dis. J. 1996,15:(6), p486-494.

12. Gill CJ, Lau J, Gorbach SL, et al. Diagnostic accuracy of 
stool assays for inflammatory bacterial gastroenteritis in developed and resource-poor countries. Clin Infect Dis. 2003; 37(3):365-375.

13. Huicho L, Sanchez D, Contreras M, et al. Occult blood and fecal leukocytes as screening test in childhood infectious diarrhea: an old problem revisited. Pediartr Infect Dis J 1993; 12:474-577

14. Lozano JM, Granados CM, Hernandez A, Perez H, Saavedra A. Uso y abuso del examen directo de materia fecal en el manejo de casos de diarrea aguda en tres instituciones de Bogotá. Actualiza Pediatr 1997; 7:152-157

15. Klein EJ, Boster DR, Stapp JR, et al. Diarrhea etiology in a Children's Hospital Emergency Department: a prospective cohort study. Clin Infect Dis. 2006: 1;43(7):807-813.
16. Denno D, Stapp JR, Boster D, et al. Etiology of Diarrhea in Pediatric Outpatient Settings. Pediatr Infect Dis J 2005; 24: $142-148$

17. Gil AB, Toledo ME, Rodríguez F. La economía de la salud, la eficiencia y el costo de oportunidad. Rev Cubana Med Gen Integr 2001; 17(4):395-8.

18. Briceño G. D.,Vera J. F., Vélez S.,Villegas S., Tovar M. B., López C., Céspedes J. Manejo de diarrea aguda de origen bacteriano: estudio de casos y controles. Rev.Medica.Sanitas 2010: 13 (3):40-50

19. Murillo OA, Leal AL, Eslava JH. Uso de Antibióticos en Infección de Vías Urinarias en una Unidad de Primer Nivel de Atención en Salud, Bogotá, Colombia. Rev. salud pública [revista en la Internet]. 2006; 8(2): 170-181. Disponible en: http://www.scielo.org.co/scielo.php?script=sci_arttext\&pid=S01240064200 